\title{
Temporal changes in allele frequency, genetic variation and inbreeding depression in small populations of the guppy, Poecilia reticulata
}

\author{
TAKAHITO SHIKANO*, TAKASHI CHIYOKUBO \& NOBUHIKO TANIGUCHI \\ Graduate School of Agricultural Science, Tohoku University, 1-1 Tsutsumidori-amamiyamachi, Sendai, \\ Miyagi 981-8555, Japan
}

\begin{abstract}
We established three closed lines of $N=10$ for the guppy Poecilia reticulata, to evaluate the relationships among temporal changes in allele frequency, genetic variation and inbreeding depression for a fitness-related trait in small populations. Genetic variation at the allozyme loci, expressed by the proportion of polymorphic loci, number of alleles per locus and heterozygosity, decreased somewhat in two closed lines but it increased in one closed line over six generations. Effective population size $(\mathrm{Ne})$ at each generation was estimated from the standardized variance in the allele frequencies. The average $N e$ was $24.4,10.3$ and 10.0 in the three closed lines. The inbreeding coefficient calculated from the $N e$ increased to $0.186,0.321$ and 0.414 , respectively. As an index of the amount of inbreeding depression, changes in salinity tolerance were examined, because this trait is strongly sensitive to inbreeding depression and decreases linearly with an increase in inbreeding coefficient. The mean value of the salinity tolerance significantly decreased to $82.5 \%, 71.7 \%$ and $67.6 \%$ in the three closed lines during the six generations, suggesting inbreeding depression for salinity tolerance. Although a significant correlation was not observed between the amount of inbreeding depression and the genetic variation, the amount of inbreeding depression correlated with the inbreeding coefficient calculated from $\mathrm{Ne}$. The regression line indicated an $8.4 \%$ decrease in the mean per $10 \%$ increase in the inbreeding coefficient and was similar to that obtained directly from full-sib matings. These results indicate that the temporal changes in the allele frequencies can provide an estimation of the amount of inbreeding depression during successive generations in small populations.
\end{abstract}

Keywords: allozyme, effective population size, genetic variation, guppy, inbreeding coefficient, inbreeding depression.

\section{Introduction}

Management of small populations may require detailed knowledge about their genetic status because such information can be of significant importance for the proper design of conservation programmes and the successful maintenance of the population. Effective population size $(\mathrm{Ne})$ is one of the important parameters that determine the population dynamics of genes. Genetic stochasticity has the effect that a decrease in $\mathrm{Ne}$ increases the rate both of inbreeding and of genetic drift (Falconer, 1989).

In small populations, inbreeding can occur as a random event due to a limited number of breeding individuals. The rate of increase per generation, $\Delta F$, is given by $\Delta F=1 / 2 N e$, where $F$ is the inbreeding coefficient, which is

*Correspondence. E-mail: shikano@bios.tohoku.ac.jp (C) 2001 The Genetics Society of Great Britain. the probability that the two genes at any locus in an individual are identical by descent (Falconer, 1989). Inbreeding causes a loss of heterozygosity with no expected change in allele frequencies. Whenever deleterious alleles are at least partially recessive, inbreeding exposes these alleles in homozygotes and causes a decrease in the mean fitness of individuals within the population (Falconer, 1989). Such effects could cumulatively erode mean population fitness, and thus increase the probability of population extinction. In contrast to inbreeding, genetic drift causes the random loss of alleles from small populations. As alleles are lost, homozygosity necessarily increases. Within any given small population, mean fitness may increase or decrease, depending on whether deleterious or advantageous alleles are lost by drift. However, averaged over a series of small populations derived from same parent population, the mean population fitness is likely to decrease due to the fixation 
of deleterious recessive alleles at some loci. Thus, inbreeding and genetic drift in small populations cause a decrease in the mean population fitness (Lande, 1988, 1994; Leberg, 1990; Mills \& Smouse, 1994; 1995b; Frankham, 1995a; Newman \& Pilson, 1997; Saccheri et al., 1998).

Protein electrophoresis has been widely used to estimate levels of genetic variation within populations and species. Low levels of heterozygosity in a population have been interpreted to be the result of a loss of genetic variation in previous bottlenecks (Allendorf \& Phelps, 1980; Ryman \& Ståhl, 1980; Taniguchi et al., 1983; Agnèse et al., 1995; Eldridge et al., 1999; Frankham, 1998). Because allozyme heterozygosity is often assumed to reflect overall genetic variability, it has also been suggested that heterozygosity estimates be used in making decisions about the management of populations and species. On the other hand, several investigators have explored the possibility of estimating $N e$ by measuring temporal changes in the allele frequency (Wright, 1948; Krimbas \& Tsakas, 1971; Nei \& Tajima, 1981; Pollak, 1983; Crow \& Denniston, 1988; Waples, 1989; Caballero, 1994; Luikart et al., 1999). The logic for this approach is that the drift variance of allele frequency between generations is $P(1-P) /(2 N e)$, where $P$ is the population frequency in the initial generation. The effects of initial allele frequency can be compensated for by using some variation of Wright's standardized variance, and this approach has formed the basis for several efforts to relate $\mathrm{Ne}$ to observed changes in allele frequencies. Waples (1989) presented a generalized form for the estimates of $\mathrm{Ne}$ from temporal changes in the allele frequencies and predicted that the method would be especially useful for small populations. Luikart et al. (1999) improved the model and derived an equation that substantially reduces three causes of the bias: the loss of alleles, the assumption that an increase in the variance in allele frequencies with time is additive (instead of multiplicative) and the assumption that selfing can occur when it cannot.

Inbreeding leads to a reduction in fitness (Falconer, 1989). If inbreeding level in small populations can be precisely measured using a genetic marker, it will be possible to expect a level for the adaptive potential and the risk of extinction of the populations. For this point of view, it is important to clarify the relationships among the temporal changes in the allele frequency, genetic variation and fitness-related traits during successive generations in small populations. However, there have been few studies of experimentally bottlenecked populations to ascertain their relationships. As a model organism for genetic analysis, the guppy Poecilia reticulata is one of the most useful teleosts because of its short life cycle, ease of breeding and establishment of populations in the laboratory and the availability of many strains (Macaranas \& Fujio, 1987; Barinova et al., 1997; Shikano \& Fujio, 1997). These strains have various characteristics in terms of morphological and physiological traits (Macaranas \& Fujio, 1987, 1990; Fujio et al., 1990; Kanda et al., 1991; Shikano \& Fujio, 1994; Ando et al., 1995). Salinity tolerance is one of the most important fitness-related traits in fish. Using fullsib matings, Shikano et al. (2000a) demonstrated that salinity tolerance is strongly sensitive to inbreeding depression and shows a linear decrease with an increase in inbreeding coefficient. Shikano et al. (1997) also reported that $F_{1}$ hybrids between strains show significant heterosis for salinity tolerance. Therefore, they proposed that the trait is one of the most useful indicators for examining the amount of inbreeding depression in populations and heterosis in crosses.

In the present study, we established three closed lines of $N=10$ for the guppy to evaluate the relationships between temporal changes in the allele frequency, genetic variation, and inbreeding depression for salinity tolerance in small populations. On the basis of the results obtained, we proposed a method to estimate the amount of inbreeding depression in small populations using allozyme markers.

\section{Materials and methods}

\section{Animals}

Three wild populations, $\mathrm{O} 1, \mathrm{O} 2$ and I, were used in this study. The $\mathrm{O} 1$ and $\mathrm{O} 2$ populations were caught in different streams on Okinawa Island and the I population in Ishigaki Island in Japan in 1996. About 30 individuals were transferred from each site to our laboratory and maintained in a $60-\mathrm{L}$ aquarium. To reduce any environmental effects which they had experienced in nature, experiments were performed using their offspring after one or two generations. Fish were fed twice daily with ground carp pellets and dried Daphnia as a supplementary diet. Each population was maintained at a temperature of $23 \pm 2^{\circ} \mathrm{C}$ with lighting for $10 \mathrm{~h}$ per day.

\section{Closed line}

Closed lines of $N=10$ were established from the $\mathrm{O} 1, \mathrm{O} 2$ and I populations. The base generation (generation 0) was established from five pairs in each population. The three closed lines were maintained in $60-\mathrm{L}$ aquaria using five pairs of parental fish per generation over six generations. Allele frequencies at the allozyme loci and salinity tolerance were examined in the base, and first to sixth generations. 


\section{Allozyme electrophoresis}

Allozyme electrophoretic and staining procedures were according to Fujio (1985). The 15 enzymic proteins surveyed were as follows: aspartate aminotransferase (Aat; E.C. 2.6.1.1), adenylate kinase (Ak; E.C. 2.7.4.3), $\alpha$-glycerophosphate dehydrogenase ( $\alpha$-Gpd; E.C. 1.1.1.8), fumarase (Fh; E.C. 4.2.1.2), glucosephosphate isomerase (Gpi; E.C. 5.3.1.9), isocitrate dehydrogenase (Idh; E.C. 1.1.1.42), lactate dehydrogenase (Ldh; E.C. 1.1.1.27), malate dehydrogenase (Mdh; E.C. 1.1.1.37), malic enzyme (Me; E.C. 1.1.1.40), mannosephosphate isomerase (Mpi; E.C. 5.3.1.8), octanol dehydrogenase (Odh; E.C. 1.1.1.73), 6-phosphogluconate dehydrogenase (6-Pgd; E.C. 1.1.1.44), phosphoglucomutase (Pgm; E.C. 5.4.2.2), sorbitol dehydrogenase (Sdh; E.C. 1.1.1.14) and superoxide dismutase (Sod; E.C. 1.15.1.1).

\section{Estimation of effective population size}

The standardized variance in the allele frequencies $\left(F_{k}\right)$ for each locus was calculated using the following equation (Krimbas \& Tsakas, 1971; Pollak, 1983):

$F_{k}=\frac{1}{A} 1 \sum_{i=1}^{A} \frac{\left(x_{i} \quad y_{i}\right)^{2}}{\left(x_{i}+y_{i}\right) / 2}$,

where $A$ is the number of alleles and $x_{i}$ and $y_{i}$ are the frequencies of the $i$ th allele in the closed line at the base generation and generation $t$, respectively, and where $t$ is the duration of the closed line in generations. Data for multiple gene loci with a varying number of alleles ( $A_{j}$ alleles at the $j$ th locus) are available. For each closed line, mean estimates of $F_{k}$ across the loci were calculated using the following equation (Waples, 1989):

Mean $F_{k}=\sum\left(\begin{array}{ll}A_{j} & 1\end{array}\right) F_{k j} / \sum\left(\begin{array}{ll}A_{j} & 1\end{array}\right)$.

To minimize the bias in the $N e$ estimates, the mean $F_{k}$ was calculated using the highly polymorphic loci (Luikart et al., 1999). These mean $F_{k}$ estimates were then used to estimate $\mathrm{Ne}$ from the following equation (Luikart et al., 1999):

$N_{e}=1 /\left[2\left(1 \quad e^{a}\right)\right] \quad 0.5$,

where $a=\ln \left[1-F_{k}-1 /\left(2 S_{0}\right)-1 /\left(2 S_{t}\right)\right] / t$, and $S_{0}$ and $S_{t}$ are the sample sizes of the individuals.

\section{Calculation of inbreeding coefficient}

Based on the genetic theory that the rate of inbreeding depends on $\mathrm{Ne}$, and thus, the variance of the changes in allele frequencies (Falconer, 1989), inbreeding coefficient
$(F)$ of each closed line was calculated using the equation (Falconer, 1989) $F_{t}=\Delta F+(1-\Delta F) F_{t-1}$, where $\Delta F=1 / 2 N e$ and $N e$ is the estimate mentioned above.

\section{Salinity tolerance}

Salinity tolerance was measured by the survival time after transfer from fresh water to $35 \mathrm{ppt}$ seawater. Mature fish, older than about 60 days, were collected from each line. Up to 20 individuals were held in a $2.5-\mathrm{L}$ aquarium filled with $35 \mathrm{ppt}$ artificial seawater (Aquasalz, Nissei, Japan) at $23.0 \pm 0.5^{\circ} \mathrm{C}$. Dead fish were recorded at $30 \mathrm{~min}$ intervals after the transfer. The fish were immediately stored at $-20^{\circ} \mathrm{C}$ for electrophoretic analysis.

\section{Statistical analysis}

Statistical comparison for salinity tolerance was assessed using one-way factorial analysis of variance (ANOVA) following by Fisher's protected least significant difference (PLSD) multiple comparison test.

\section{Results}

\section{Genetic variation}

Electrophoretic analyses revealed five polymorphic loci (Aat-1:2, Pgm-2:3, Gpi-1:2, Gpi-2:2 and Sod-2:2; the first number denotes the locus and the second refers to the number of alleles found at this locus). The 23 loci which were monomorphic for the same allele were Aat-2, Aat-3, Ak, Fh, $\alpha-G p d-1, \alpha-G p d-2, G p i-1, I d h-1, I d h-2, L d h-1$, Ldh-2, Ldh-3, Mdh-1, Mdh-2, Mdh-3, Me, Mpi, Odh-1, Odh-2, 6-Pgd, Sdh-1, Sdh-2 and Sod-1. The banding patterns of Aat-1, Pgm-2, Gpi-1, Gpi-2 and Sod-2 could be interpreted in terms of Mendelian loci in agreement with the allozyme data from Macaranas \& Fujio (1987). Table 1 shows the allele frequencies in three closed lines over six generations. Genetic variation was examined using the proportion of polymorphic loci, the number of alleles per locus and the average heterozygosity (Table 2). These values did not show regular decreases with the generation time in all three closed lines. The average heterozygosity decreased somewhat in the $\mathrm{O} 1$ and I closed lines but it increased in the $\mathrm{O} 2$ closed line. The proportion of homozygotes relative to the base generation $\left(1-H_{t} / H_{0}\right)$ was positive in the $\mathrm{O} 1$ and $\mathrm{I}$ closed lines and negative in the $\mathrm{O} 2$ closed lines.

\section{Estimation of $\mathrm{Ne}$ and $\mathrm{F}$}

Estimates of $N e$ using the mean $F_{k}$ values are presented in Table 3. The mean $F_{k}$ was calculated using the highly 
Table 1 Temporal changes in allele frequencies at five polymorphic allozyme loci in three closed lines of guppies

\begin{tabular}{|c|c|c|c|c|c|c|c|c|c|c|c|c|c|c|}
\hline \multirow[b]{2}{*}{ Line } & \multirow[b]{2}{*}{ Gen. } & \multirow[b]{2}{*}{$N$} & \multicolumn{2}{|c|}{ Aat-1 } & \multicolumn{3}{|c|}{ Gpi-2 } & \multicolumn{2}{|c|}{ Pgm-1 } & \multicolumn{3}{|c|}{ Pgm-2 } & \multicolumn{2}{|c|}{ Sod-2 } \\
\hline & & & $A$ & $B$ & $A$ & $B$ & C & $A$ & $B$ & $A^{\prime}$ & $A$ & $B$ & $A$ & $B$ \\
\hline \multirow[t]{7}{*}{$\mathrm{O} 1$} & 0 & 50 & 0.520 & 0.480 & .110 & 0.830 & 0.060 & 0.150 & 0.850 & 0.070 & 0.880 & 0.050 & 0.320 & 0.680 \\
\hline & 1 & 31 & 0.565 & 0.435 & 0.129 & 0.855 & 0.016 & 0.161 & 0.839 & 0.161 & 0.823 & 0.016 & 0.145 & 0.855 \\
\hline & 2 & 74 & 0.595 & 0.405 & 0.095 & 0.810 & 0.095 & 0.203 & 0.797 & 0.176 & 0.804 & 0.020 & 0.122 & 0.878 \\
\hline & 3 & 75 & 0.671 & 0.329 & 0.062 & 0.938 & 0.000 & 0.171 & 0.829 & 0.167 & 0.800 & 0.033 & 0.227 & 0.773 \\
\hline & 4 & 35 & 0.757 & 0.243 & 0.043 & 0.914 & 0.043 & 0.214 & 0.786 & 0.200 & 0.800 & 0.000 & 0.214 & 0.786 \\
\hline & 5 & 46 & 0.663 & 0.337 & 0.022 & 0.924 & 0.054 & 0.087 & 0.913 & 0.098 & 0.891 & 0.011 & 0.304 & 0.696 \\
\hline & 6 & 98 & 0.699 & 0.301 & 0.051 & 0.852 & 0.097 & 0.092 & 0.908 & 0.087 & 0.821 & 0.092 & 0.337 & 0.663 \\
\hline \multirow[t]{7}{*}{$\mathrm{O} 2$} & 0 & 51 & 0.873 & 0.127 & 0.206 & 0.794 & 0.000 & 0.147 & 0.853 & 0.000 & 0.735 & 0.265 & 0.000 & 1.000 \\
\hline & 1 & 40 & 0.913 & 0.087 & 0.225 & 0.775 & 0.000 & 0.000 & 1.000 & 0.000 & 0.750 & 0.250 & 0.038 & 0.962 \\
\hline & 2 & 47 & 0.840 & 0.160 & 0.128 & 0.872 & 0.000 & 0.032 & 0.968 & 0.032 & 0.660 & 0.308 & 0.000 & 1.000 \\
\hline & 3 & 73 & 0.671 & 0.329 & 0.062 & 0.938 & 0.000 & 0.171 & 0.829 & 0.171 & 0.610 & 0.219 & 0.185 & 0.815 \\
\hline & 4 & 31 & 0.694 & 0.306 & 0.081 & 0.871 & 0.048 & 0.242 & 0.758 & 0.210 & 0.742 & 0.048 & 0.161 & 0.839 \\
\hline & 5 & 45 & 0.733 & 0.267 & 0.022 & 0.956 & 0.022 & 0.256 & 0.744 & 0.278 & 0.555 & 0.167 & 0.144 & 0.856 \\
\hline & 6 & 37 & 0.811 & 0.189 & 0.176 & 0.824 & 0.000 & 0.203 & 0.797 & 0.216 & 0.581 & 0.203 & 0.027 & 0.973 \\
\hline \multirow[t]{7}{*}{ I } & 0 & 50 & 0.670 & 0.330 & 0.020 & 0.650 & 0.330 & 0.410 & 0.590 & 0.000 & 0.320 & 0.680 & 0.000 & 1.000 \\
\hline & 1 & 37 & 0.838 & 0.162 & 0.054 & 0.797 & 0.149 & 0.000 & 1.000 & 0.000 & 0.622 & 0.378 & 0.027 & 0.973 \\
\hline & 2 & 78 & 0.724 & 0.276 & 0.000 & 0.641 & 0.359 & 0.000 & 1.000 & 0.000 & 0.615 & 0.385 & 0.000 & 1.000 \\
\hline & 3 & 55 & 0.836 & 0.164 & 0.000 & 0.627 & 0.373 & 0.000 & 1.000 & 0.000 & 0.645 & 0.355 & 0.000 & 1.000 \\
\hline & 4 & 35 & 0.814 & 0.186 & 0.000 & 0.529 & 0.471 & 0.000 & 1.000 & 0.000 & 0.529 & 0.471 & 0.329 & 0.671 \\
\hline & 5 & 36 & 0.542 & 0.458 & 0.000 & 0.681 & 0.319 & & 1.000 & 0.000 & 0.639 & 0.361 & 0.042 & 0.958 \\
\hline & 6 & 94 & 0.777 & 0.223 & 0.000 & 0.755 & 0.245 & 0.000 & 1.000 & 0.000 & 0.707 & 0.293 & 0.000 & 1.000 \\
\hline
\end{tabular}

\begin{tabular}{ccccccc}
\hline Line & Gen. & $N$ & $\begin{array}{c}\text { Proportion of } \\
\text { polymorphic loci }\end{array}$ & $\begin{array}{c}\text { Number of } \\
\text { alleles/locus }\end{array}$ & $\begin{array}{c}\text { Average } \\
\text { heterozygosity }\end{array}$ & $1-H_{t} / H_{0}$ \\
\hline O1 & 0 & 50 & 0.178 & 1.25 & 0.061 & 0 \\
& 1 & 31 & 0.178 & 1.18 & 0.056 & +0.082 \\
& 2 & 74 & 0.178 & 1.21 & 0.060 & +0.016 \\
& 3 & 75 & 0.178 & 1.18 & 0.054 & +0.115 \\
& 4 & 35 & 0.178 & 1.14 & 0.054 & +0.115 \\
& 5 & 46 & 0.178 & 1.18 & 0.049 & +0.197 \\
& 6 & 98 & 0.178 & 1.25 & 0.057 & +0.049 \\
$\mathrm{O} 2$ & 0 & 51 & 0.143 & 1.14 & 0.042 & 0 \\
& 1 & 40 & 0.107 & 1.11 & 0.034 & +0.190 \\
& 2 & 47 & 0.107 & 1.11 & 0.037 & +0.119 \\
& 3 & 73 & 0.178 & 1.21 & 0.061 & -0.452 \\
& 4 & 31 & 0.178 & 1.18 & 0.061 & -0.452 \\
& 5 & 45 & 0.143 & 1.18 & 0.060 & -0.429 \\
& 6 & 37 & 0.143 & 1.18 & 0.055 & -0.310 \\
& 0 & 50 & 0.143 & 1.14 & 0.065 & 0 \\
& 1 & 37 & 0.107 & 1.14 & 0.041 & +0.369 \\
& 2 & 78 & 0.107 & 1.11 & 0.048 & +0.262 \\
& 3 & 55 & 0.107 & 1.11 & 0.043 & +0.338 \\
& 4 & 35 & 0.143 & 1.14 & 0.062 & +0.046 \\
& 5 & 36 & 0.107 & 1.11 & 0.053 & +0.185 \\
6 & 94 & 0.107 & 1.11 & 0.040 & +0.385 \\
\hline
\end{tabular}

Table 2 Temporal changes in genetic variation in three closed lines of guppies

$H_{0}$ and $H_{t}$ represent average heterozygosities at generation 0 and $t$, respectively.

$1-H_{t} / H_{0}$ refers to increase in homozygosity with respect to the base generation. 
Table 3 Estimates of effective population size $(\mathrm{Ne})$ and inbreeding coefficient $(F)$ in three closed lines of guppies

\begin{tabular}{lccrcc}
\hline Line & Gen. & Mean $F_{k}$ & \multicolumn{1}{c}{$N e$} & $\Delta F$ & $F$ \\
\hline O1 & 1 & 0.0494 & 6.1 & 0.082 & 0.082 \\
& 2 & 0.0589 & 12.5 & 0.040 & 0.119 \\
& 3 & 0.0565 & 19.5 & 0.026 & 0.141 \\
& 4 & 0.0905 & 16.2 & 0.031 & 0.168 \\
& 5 & 0.0445 & 36.8 & 0.014 & 0.179 \\
& 6 & 0.0373 & 55.4 & 0.009 & 0.186 \\
O2 & 1 & 0.0843 & 4.2 & 0.119 & 0.119 \\
& 2 & 0.0587 & 11.9 & 0.042 & 0.156 \\
& 3 & 0.1653 & 7.2 & 0.069 & 0.215 \\
& 4 & 0.1966 & 7.7 & 0.065 & 0.266 \\
& 5 & 0.2032 & 9.6 & 0.052 & 0.304 \\
& 6 & 0.1082 & 21.0 & 0.024 & 0.321 \\
$\mathrm{I}$ & 1 & 0.1790 & 2.0 & 0.254 & 0.254 \\
& 2 & 0.1015 & 7.7 & 0.065 & 0.302 \\
& 3 & 0.1543 & 7.6 & 0.066 & 0.348 \\
& 4 & 0.1004 & 14.8 & 0.034 & 0.370 \\
& 5 & 0.1295 & 14.8 & 0.034 & 0.391 \\
& 6 & 0.1844 & 13.2 & 0.038 & 0.414 \\
\hline
\end{tabular}

polymorphic loci over the six generations in each closed line (Aat-1, Gpi-2, Pgm-1, Pgm-2 and Sod-2 in the O1 population; Aat-1, Gpi-2, Pgm-1 and Pgm-2 in the O2 population; Aat-1, Gpi-2 and Pgm-2 in the I population). The $N e$ at each generation ranged from 6.1 to 55.4 with a mean of $24.4(\mathrm{O} 1)$, from 4.2 to 21.0 with a mean of $10.3(\mathrm{O} 2)$ and from 2.0 to 14.8 with a mean of $10.0(\mathrm{I})$. The rate of inbreeding per generation $(\Delta F)$ calculated from the $N e$ ranged from 0.009 to 0.082 (O1), from 0.024 to $0.119(\mathrm{O} 2)$ and from 0.034 to $0.254(\mathrm{I})$ while inbreeding coefficient $(F)$ increased to $0.186(\mathrm{O} 1), 0.321$ $(\mathrm{O} 2)$ and 0.414 (I) during the six generations.

\section{Relationship between salinity tolerance and $\mathrm{F}$}

Table 4 shows changes in the survival times in $35 \mathrm{ppt}$ seawater for the three closed lines. The mean survival time significantly decreased after one or two generations $(P<0.05$ or $P<0.01)$. In comparison with the base generation, the mean value decreased to $82.5 \%, 71.7 \%$ and $67.6 \%$ after the six generations in the $\mathrm{O} 1, \mathrm{O} 2$ and $\mathrm{I}$ closed lines, respectively. The standard deviations also decreased in all three closed lines.

The mean survival time in $35 \mathrm{ppt}$ seawater did not correlate with the increased proportion of homozygotes $\left(1-H_{t} / H_{0}\right)$ and the average heterozygosity $(P>0.05)$ but it significantly correlated with the inbreeding coefficient calculated from $N e$, in all three closed lines $(P<0.05$ or $P<0.01)$ (Table 5). A significant negative correlation was also observed between the inbreeding
Table 4 Temporal changes in survival times in $35 \mathrm{ppt}$ seawater in three closed lines of guppies

\begin{tabular}{ccccc}
\hline Line & Gen. & $N$ & Mean (h) \pm SD & $\begin{array}{c}\text { Relative } \\
\text { value }(\%)\end{array}$ \\
\hline O1 & 0 & 50 & $4.96 \pm 1.70$ & 100.0 \\
& 1 & 31 & $4.66 \pm 1.16$ & 94.0 \\
& 2 & 74 & $4.47 \pm 1.30^{*}$ & 90.1 \\
& 3 & 75 & $3.95 \pm 1.22^{* *}$ & 79.6 \\
& 4 & 35 & $4.27 \pm 1.23^{*}$ & 86.1 \\
& 5 & 46 & $4.37 \pm 1.14^{*}$ & 88.1 \\
& 6 & 98 & $4.09 \pm 1.12^{* *}$ & 82.5 \\
O2 & 0 & 51 & $5.58 \pm 1.73$ & 100.0 \\
& 1 & 40 & $4.46 \pm 1.61^{* *}$ & 79.9 \\
& 2 & 47 & $4.54 \pm 1.35^{* *}$ & 81.4 \\
& 3 & 73 & $4.21 \pm 1.20^{* *}$ & 75.4 \\
& 4 & 31 & $4.02 \pm 1.17^{* *}$ & 72.0 \\
& 5 & 45 & $3.56 \pm 1.31^{* *}$ & 63.8 \\
& 6 & 37 & $4.00 \pm 1.10^{* *}$ & 71.7 \\
& 0 & 50 & $4.97 \pm 1.09$ & 100.0 \\
& 1 & 37 & $3.70 \pm 0.97^{* *}$ & 74.4 \\
& 2 & 78 & $3.25 \pm 0.74^{* *}$ & 65.4 \\
& 3 & 55 & $3.31 \pm 0.78^{* *}$ & 66.6 \\
& 4 & 35 & $3.41 \pm 0.83^{* *}$ & 68.6 \\
& 5 & 36 & $3.71 \pm 0.63^{* *}$ & 74.6 \\
& 6 & 94 & $3.39 \pm 0.63^{* *}$ & 67.6 \\
\hline
\end{tabular}

Significantly different from the value at generation 0 with $* P<0.05$ and ${ }^{* *} P<0.01$.

Table 5 Regression analysis of the survival time in $35 \mathrm{ppt}$ seawater on the inbreeding coefficient calculated from the $\mathrm{Ne}$ in three closed lines of guppies

\begin{tabular}{lccccc}
\hline Line & Coefficient & Intercept & $R^{2}$ & $N$ & $P$ \\
\hline O1 & -4.378 & 4.943 & 0.716 & 7 & 0.016 \\
O2 & -5.248 & 5.374 & 0.887 & 7 & 0.002 \\
I & -3.811 & 4.805 & 0.810 & 7 & 0.006 \\
\hline
\end{tabular}

coefficient and the relative value of the mean survival time within the three closed lines $(P<0.01)$, as shown in Fig. 1. The regression line indicated an $8.4 \%$ decrease in the mean per $10 \%$ increase in the inbreeding coefficient.

\section{Discussion}

Population genetic theory predicts that a decrease in $\mathrm{Ne}$ increases the rate of both inbreeding and genetic drift (Falconer, 1989). Although the effects of inbreeding and genetic drift are conceptually distinct, they are generally confounded in real populations. This is because in small panmictic populations the probabilities of both mating with a relative and losing alleles by chance are increased 


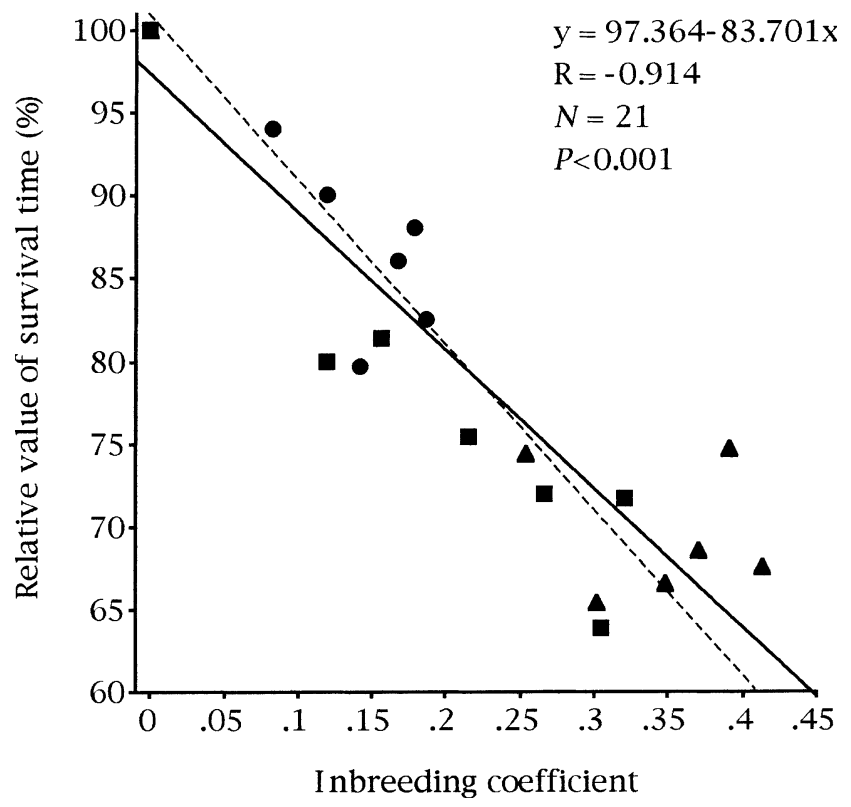

Fig. 1 Relationship between the inbreeding coefficient calculated from the $\mathrm{Ne}$ and the relative value of mean survival time in $35 \mathrm{ppt}$ seawater within three closed lines of guppies. Data are from $\mathrm{O} 1(\boldsymbol{O}), \mathrm{O} 2(\boldsymbol{\square})$ and $\mathrm{I}(\boldsymbol{\Delta})$ closed lines. Dotted line indicates the regression line obtained from full-sib matings (Shikano et al. 2000a).

relative to these probabilities in large panmictic populations (Ellstrand \& Elam, 1993). If the level of inbreeding, which leads to a reduction in fitness (Falconer, 1989), can be precisely measured in small panmictic populations using a genetic marker, it will be possible to predict the level of the adaptive potential and the risk of extinction of the populations. From this point of view, the present experiment was designed to study the relationships among the temporal changes in the allele frequency, genetic variation and inbreeding depression for a fitness-related trait during successive generations in small populations of the guppy.

There is considerable theoretical support for the hypothesis that a population that has passed through a bottleneck in the effective number of founding individuals should have less genetic variation than it contained prior to the bottleneck (Wright, 1931; Nei et al., 1975; Chakraborty \& Nei, 1977). For neutral markers the relationship among the decline in heterozygosity $(H), N e$ and number of generations $(t)$ is expressed by the equation $H_{t} / H_{0}=(1-1 / 2 N e)^{t}$, where $H_{0}$ is the original heterozygosity and $H_{t}$ is heterozygosity for generation $t$ (Crow \& Kimura, 1970). Based on this equation, heterozygosities should decrease from 0.061 to 0.045 , from 0.042 to 0.031 and from 0.065 to 0.048 after six generations in the $\mathrm{O} 1, \mathrm{O} 2$ and I closed lines of $N=10$, respectively, with the assumption of random mating. However, the present results showed that the heterozygosities in the three closed lines fluctuated during successive generations and were not in agreement with the expected values. Therefore, the inbreeding coefficient estimated from the equation $F=1-H_{t} / H_{0}$. (Falconer, 1989) is not applicable. Evidence from experimental populations of mosquitofish, Gambusia holbrooki, initiated from different numbers of founder individuals (Leberg, 1992), demonstrated that heterozygosity per se may be a poor indicator of population bottlenecks due to an increase in the variance of heterozygosity arising from drift of allele frequencies. Based on theoretical considerations, sampling only a few polymorphic loci would make it difficult to detect the effects of bottlenecks on levels of heterozygosity. The number of polymorphic loci and alleles observed in the present study is only five loci and two to three alleles. These small numbers suggest that sampling error may result in poor estimates of the overall heterozygosity in the bottlenecked populations.

The present study experimentally demonstrated that the allele frequencies randomly fluctuated from generation to generation as a result of the finite sampling of gametes in small populations of the guppy. We estimated $\mathrm{Ne}$ from the temporal changes in the allele frequencies using the method of Luikart et al. (1999). Although the $N e$ fluctuated among generations, the mean values are the same level with the actual number of breeding individuals. The fluctuation may be in part due to different numbers of males and females and nonrandom distribution of family size (Falconer, 1989). We also estimated $\mathrm{Ne}$ using the following equation reported by Nei \& Tajima (1981):

$N_{e}=t /\left\{2\left[\begin{array}{lll}F_{k} & 1 /\left(2 S_{0}\right) & 1 /\left(2 S_{t}\right)\end{array}\right]\right\}$.

The Ne estimated ranged from 21.5 to 134.9 with a mean of $59.0(\mathrm{O} 1)$, from 8.1 to 35.4 with a mean of $17.5(\mathrm{O} 2)$ and from 3.2 to 26.3 with a mean of 15.6 (I), indicating that this method caused overestimates against the actual number of breeding individuals and higher fluctuation than that observed in estimates using the method of Luikart et al. (1999). As indicated using simulation models, the present study experimentally demonstrated that the method of Luikart et al. (1999) may be more useful for estimates of $\mathrm{Ne}$ and detection of bottlenecks in small populations than that of Nei \& Tajima (1981).

Shikano et al. (2000a) indicated that salinity tolerance is one of the most useful indicators for examining the amount of inbreeding depression in small populations of the guppy, because the trait is strongly sensitive to inbreeding depression and heterosis and shows a linear decrease with an increase in inbreeding coefficient. The linear relationship between salinity tolerance and 
inbreeding coefficient suggests that inbreeding depression for salinity tolerance is caused by additive gene action among the loci responsible for inbreeding depression (Shikano et al. 2000a), as similarly reported in various fitness-related traits of animals and plants (Falconer, 1989; Hauser \& Loeschcke, 1995; Analla et al., 1999). Thus, the use of salinity tolerance as an index of inbreeding depression allowed us to obtain precise information about the changes in the amount of inbreeding depression during successive generations in the closed lines. The present study showed that the mean and variance of the salinity tolerance significantly decreased in all the three closed lines. The characterization is in agreement with the result obtained in full-sib matings (Shikano et al. 2000a), suggesting inbreeding depression for salinity tolerance in the closed lines. Based on the fact that the mean value of salinity tolerance decreases at a rate of $9.1 \%$ per $10 \%$ increase in the inbreeding coefficient (Shikano et al. 2000a), the decrease in the mean value during the six generations corresponds to the inbreeding coefficients of $0.192,0.311$ and 0.356 in the $\mathrm{O} 1, \mathrm{O} 2$ and I closed lines, respectively.

The most important result in our study is that the amount of depression in salinity tolerance was significantly correlated with the inbreeding coefficient estimated from the temporal changes in the allele frequencies during successive generations in the three closed lines. The regression line indicating an $8.4 \%$ decrease in the mean per $10 \%$ increase in the inbreeding coefficient is similar to that directly obtained from the full-sib matings as shown in Fig. 1 (Shikano et al. 2000a). These results suggest that the temporal changes in the allele frequencies can provide estimates of $\mathrm{Ne}$ that are available to predict inbreeding depression in small populations. On the other hand, the amount of depression in salinity tolerance did not correlate with the genetic variation and the inbreeding coefficient estimated from the equation $F=1-H_{t} / H_{0}$. Several investigators have reported significant associations between the allozyme heterozygosity and fitness-related traits (Mitton \& Grant, 1984; Zouros \& Foltz, 1987). Our previous study also showed that domestic strains of the guppy showed lower genetic variation at the allozyme loci and salinity tolerance than did wild populations (Shikano et al. 2000b). It is possible that inbreeding depression can occur before a decrease in the genetic variation is detected during successive generations in small populations. This may be due to the fact that small number of polymorphic loci and alleles result in poor estimates of overall heterozygosity, especially for earlier generations of small populations. Therefore, temporal changes in genetic variation cannot be used to estimate inbreeding depression if the number of polymorphic loci and alleles examined is small.

(c) The Genetics Society of Great Britain, Heredity, 86, 153-160.
The temporal method and the guideline provided here should be greatly useful for conservation biology and the management of small populations where small effective population sizes may increase the effect of inbreeding depression.

\section{Acknowledgements}

This work was partly supported by a Grant-in-Aid for Scientific Research from the Ministry of Education, Science, Sports and Culture of Japan (00111152). T.S. was supported by Research Fellowships of the Japan Society for the Promotion of Science for Young Scientists.

\section{References}

AGNÈSE, J. F., OTEME, Z. J. AND GILLES, S. 1995. Effects of domestication on genetic variability, fertility, survival and growth rate in a tropical siluriform: Heterobranchus longifilis Valenciennes 1840. Aquaculture, 131, 197-204.

ALLENDORF, F. W. AND PHELPS, S. R. 1980. Loss of genetic variation in a hatchery stock of cutthroat trout. Trans. Am. Fish. Soc., 109, 537-543.

ANAlla, M., MONTILla, J. M. AND SerRadilla, J. M. 1999. Study of the variability of the response to inbreeding for meat production in Merino sheep. J. Anim. Breed. Genet., 116, 481-488.

ANDO, D., NAKAJIMA, M. AND FUJIO, Y. 1995. Strain differences of vertebral abnormality in the guppy Poecilia reticulata. Tohoku J. Agric. Res., 46, 29-34.

BARINOVA, A. A., NAKAJIMA, M. AND FUJIO, Y. 1997. Genetic differentiation of laboratory populations in the guppy Poecilia reticulata. Fish Genet. Breed. Sci., 25, 19-26.

CABAllero, A. 1994. Developments in the prediction of effective population size. Heredity, 73, 657-679.

CHAKRABORTY, R. AND NEI, M. 1977. Bottleneck effects on average heterozygosity and genetic distance with the stepwise mutation model. Evolution, 31, 347-356.

CROW, J. AND DENNISTON, C. 1988. Inbreeding and variance effective population numbers. Evolution, 42, 482-495.

Crow, J. F. AND KIMURA, M. 1970. An Introduction to Population Genetics Theory. Harper \& Row, New York.

ELDRIDGE, M. D. B., KING, J. M., LOUPIS, A. K., SPENCER, P. B. S. ET AL. 1999. Unprecedented low levels of genetic variation and inbreeding depression in an island population of the black-footed rock-wallaby. Conserv. Biol., 13, 531-541.

ELlSTRAND, N. AND ElAM, D. 1993. Population genetic consequences of small population size: Implication for plant conservation. Ann. Rev. Ecol. Syst., 24, 217-242.

FALCONER, D. S. 1989. Introduction to Quantitative Genetics, 3rd edn. Longman, New York.

FRANKHAM, R. 1995a. Inbreeding depression: a threshold effect. Conserv. Biol., 9, 792-799.

FrAnkham, R. 1995b. Conservation genetics. Ann. Rev. Genet., 29, 305-327. 
FRANKHAM, R. 1998. Inbreeding and extinction: Island populations. Conserv. Biol., 12, 665-675.

FUJIO, Y. 1985. Study on the genetic characteristics of fish and shellfish in isozyme analysis. In: Fujio, Y. (ed) Nosuisho Tokubetsu Shiken Houkokusyo, pp. 1-65. Nosuisho, Tokyo. FUJIO, Y., NAKAJIMA, M. AND NAGAHAMA, M. 1990. Detection of a low temperature-resistant gene in the guppy (Poecilia reticulata), with reference to sex-linked inheritance. Jap. J., Genet., 65, 201-207.

HAUSER, T. P. AND LOESCHCKE, V. 1995. Inbreeding depression in Lychnis flos-cuculi (Caryophyllaceae): Effects of different levels of inbreeding. J. Evol. Biol., 8, 589-600.

KANDA, N., NAKAJIMA, M. AND FUJIO, Y. 1991. Strain differences at thermal resistance in the guppy, Poecilia reticulata. Tohoku J. Agric. Res., 42, 25-31.

KRIMBAS, C. B. AND TSAKAS, S. 1971. The genetic of Dacus oleae $\mathrm{V}$. Changes of esterase polymorphism in a natural population following insecticide control - selection or drift? Evolution, 25, 454-460.

LANDE, R. 1988. Genetics and demography in biological conservation. Science, 241, 1455-1459.

LANDE, R. 1994. Risk of population extinction from fixation of new deleterious mutations. Evolution, 48, 1460-1469.

LEBERG, P. L. 1990. Influence of genetic variability on population growth: implications for conservation. J. Fish Biol., 37, 193-195.

LEBERG, P. L. 1992. Effects of population bottlenecks on genetic diversity as measured by allozyme electrophoresis. Evolution, 46, 477-495.

LUIKART, G., CORNUET, J.-M. AND ALlENDORF, F. W. 1999. Temporal changes in allele frequencies provide estimates of population bottleneck size. Conserv. Biol., 13, 523-530.

MACARANAS, J. M. AND FUJIO, Y. 1987. Genetic differences among strains of the guppy, Poecilia reticulata. Tohoku J. Agric. Res., 37, 75-85.

MACARANAS, J. M. AND FUJIO, Y. 1990. Strain differences in cultured fish - isozymes and performance traits as indicators. Aquaculture, 85, 69-82.

MILLS, S. L. AND SMOUSE, P. E. 1994. Demographic consequences of inbreeding in remnant populations. Am. Nat., 144, $412-431$.

MITTON, J. B. AND GRANT, M. C. 1984. Associations among protein heterozygosity, growth rate and developmental homeostasis. Ann. Rev. Ecol. Syst., 15, 479-499.

NEI, M. AND TAJIMA, F. 1981. Genetic drift and estimation of effective population size. Genetics, 98, 625-640.

NEI, M., MARUYAMA, T. AND CHAKRABORTY, R. 1975. The bottleneck effect and genetic variability in populations. Evolution, 29, 1-10.
NEWMAN, D. AND PILSON, D. 1997. Increased probability of extinction due to decreased genetic effective population size: Experimental populations of Clarkia pulchella. Evolution, 51, 345-362.

POLLAK, E. 1983. A new method for estimating the effective population size from allele frequency changes. Genetics, 104, 531-548.

RYMAN, N. AND STåHL, G. 1980. Genetic changes in hatchery stocks of brown trout (Salmo trutta). Can. J. Aquat. Sci., 37, $82-87$.

SACCHERI, I., KUUSSAARI, M., VIKMAN, P., FORTELIUS, W. ET AL. 1998. Inbreeding and extinction in a metapopulation. Nature, 392, 491-494.

SHIKANO, T. AND FUJIO, Y. 1994. Strain differences at salinity resistance in the guppy, Poecilia reticulata. Fish. Genet. Breed. Sci., 20, 47-53.

SHIKANO, T. AND FUJIO, Y. 1997. Successful propagation in seawater of the guppy Poecilia reticulata with reference to high salinity tolerance at birth. Fish. Sci., 63, 573-575.

SHIKANO, T., NAKADATE, M., NAKAJIMA, M. AND FUJIO, Y. 1997. Heterosis and maternal effects in salinity tolerance of the guppy Poecilia reticulata. Fish. Sci., 63, 893-896.

SHIKANO, T., CHIYOKUBO, T. AND TANIGUCHI, N. 2000a. Effect of inbreeding on salinity tolerance in the guppy (Poecilia reticulata). Aquaculture, in press.

SHIKANO, T., CHIYOKubo, T., NAKADATE, M. AND FUJIO, Y. 2000b. The relationship between allozyme heterozygosity and salinity tolerance in wild and domestic populations of the guppy (Poecilia reticulata). Aquaculture, 184, 233245.

TANIGUCHI, N., SUMANTADINATA, K. AND IYAMA, S. 1983. Genetic change in the first and second generations of hatchery stock of black seabream. Aquaculture, 35, 309-320.

WAPLES, R. S. 1989. A generalized approach for estimating effective population size from temporal changes in allele frequency. Genetics, 121, 379-391.

WRIGHT, s. 1931. Evolution in Mendelian populations. Genetics, 28, 114-138.

WRIGHT, s. 1948. On the roles of directed and random changes in gene frequency in the genetics of populations. Evolution, 2, 279-294.

zouros, E. AND FOLTZ, D. w. 1987. The use of allelic isozyme variation for the study of heterosis. In: Rattazi, M. C., Scandalios, J. G. and Whitt, G. S. (eds) Isozymes: Current Topics in Biological and Medical Research, vol. 13, pp. 1-59. Liss, New York. 